\title{
Role of Bandwidths and Energy Gap in Formation of Ground State of Ultra-Cold Bosons in Artificial Magnetic Fields
}

\author{
K. Patucha, B. Grygiel and T.A. Zaleski \\ Institute of Low Temperature and Structure Research, Polish Academy of Sciences,
}

Okólna 2, 50-422 Wrocław, Poland

\begin{abstract}
We study the properties of ultra-cold bosons in optical lattice in arbitrary gauge potentials. Using quantum rotor approach we are able to go beyond mean-field approximation thus taking into account subtleties of the band structure of the artificial magnetic field. This allows us to elucidate the interplay of the subbands widths and energy gaps on the formation of the coherent state. As a result, we are able to pinpoint the elements of the band structure, which are crucial to proper theoretical description of the synthetic magnetic field in a lattice bosonic system. This leads us finally to a method of approximation of the Hofstadter butterfly spectrum with a simpler band structure and use it to investigate the ground state of the system for a wide range of magnetic fluxes.
\end{abstract}

DOI: 10.12693/APhysPolA.130.637

PACS/topics: $67.85 . \mathrm{Hj}$, 05.30.Jp, 03.75.Lm

\section{Introduction}

Optical lattices are periodic potential created by counter-propagating laser beams $[1,2]$. They allow for trapping ultra-cold atoms in highly tunable environment, in which both hopping and interactions can be precisely controlled [3]. In case of bosons, a phase transition between superfluid (SF) and the Mott insulating (MI) state can be observed, which is a hallmark feature of strongly interacting systems.

Since atoms in optical lattices are neutral, effects of magnetic field (analogous to effect of magnetic field on electrons in condensed matter systems) must be simulated. In analogy between the Lorentz and Coriolis force [2] this can be realized using a rotation of the system. However, other methods utilizing direct imprinting of geometric phase resulting from the magnetic field on the particles are more handy: e.g. photon-assisted tunnelling $[4-8]$ or lattice-shaking $[9,10]$.

In the lattice systems, natural measure of magnetic field is a magnetic flux through elementary cell in units of magnetic flux quantum: $\alpha=p / q$. The denominator $q$ determines number of magnetic energy band. Energy spectrum of the lattice in magnetic field is given by the Hofstadter butterfly [11] which has period $\alpha=1$ and is a consequence of the interplay between periodicity of the lattice and geometric phase factor. It is a fractal structure, which changes parameters, such as total bandwidth, number of bands and flatness of the lowest band, in non-monotonic way with respect to magnetic field. This has profound influence on the ground state of interacting bosons in a lattice.

In this paper we analyze the influence of the parameters of the band structure on the phase diagram. We use the Bose-Hubbard model in quantum rotor approach [12-14] which takes into account the dependence on the internal energy structure in a non-trivial way. We performed a thorough analysis of the influence of the pa- rameters of the band structure on the ground state. This leads us to a method of simulation of magnetic densities of states.

The paper is organised as follows: first we give a brief introduction to the Bose-Hubbard model and quantum rotor approach. In the following section, we study influence of parameters of the band structure on the phase diagram. In Sect. 4, the method of simulation of magnetic densities of states is proposed. Using this method, phase diagram for wide range of magnetic flux values is calculated. Finally, we summarise our results.

\section{Model and method}

Ultra-cold bosons in optical lattice are well described by the Bose-Hubbard model $[15,16]$ :

$$
\begin{aligned}
\hat{H} & =-\sum_{\langle i, j\rangle} t_{i j}\left(\hat{a}_{i}^{\dagger} \hat{a}_{j}+\text { H.c. }\right)+\frac{U}{2} \sum_{i} \hat{n}_{i}^{2} \\
& -\bar{\mu} \sum_{i} \hat{n}_{i},
\end{aligned}
$$

where $\hat{a}_{i}\left(\hat{a}_{i}^{\dagger}\right)$ is bosonic annihilation (creation) operator and $\hat{n}_{i}$ is the particle number operator on the lattice site $i$. The first term describes hopping between the nearest neighbours with hopping integral $t_{i j}$, the next term is related to on-site repulsive interaction, while the last contains shifted chemical potential, $\bar{\mu}=\mu+U / 2$. The effects of synthetic magnetic field are introduced by the Peierls substitution [11, 17]:

$$
t_{i j} \rightarrow t_{i j} \exp \left(\frac{2 \pi \mathrm{i}}{\Phi_{0}} \int_{\boldsymbol{r}_{i}}^{\boldsymbol{r}_{j}} \boldsymbol{A} \cdot \mathrm{d} \boldsymbol{l}\right),
$$

where $\boldsymbol{A}$ is vector potential and $\Phi_{0}$ is the flux quantum (for electron systems $\Phi_{0}=e / h$ ). Since, this inclusion effectively renormalizes inter-site hopping, the general form of $t_{i j}$ can be maintained through the further calculations, making the results valid both with and without the magnetic field. 


\subsection{Method}

In order to solve the Bose-Hubbard model, while including spatial fluctuations, which are necessary to accommodate the presence of the magnetic field, quantum rotor approach is used [13]. Partition function is written in path integral formalism using complex fields $\bar{a}(\tau), a(\tau)$ :

$$
Z=\int[\mathcal{D} \bar{a} \mathcal{D} a] \mathrm{e}^{-S[\bar{a}, a]},
$$

with the action of the form

$$
S[\bar{a}, a]=\int_{0}^{\beta} \mathrm{d} \tau H(\bar{a}, a)+\int_{0}^{\beta} \mathrm{d} \tau \sum_{i} \bar{a}_{i} \frac{\partial}{\partial \tau} a_{i},
$$

where Hamiltonian $H(\bar{a}, a)$ is of the same form as (1) with creation and annihilation operators replaced by the complex fields $\bar{a}_{i}(\tau), a_{i}(\tau)$. Separating these into phase $\phi_{i}(\tau)$ and amplitude $b_{i}(\tau)$ degrees of freedom $a_{i}(\tau)=b_{i}(\tau) \exp \left(\mathrm{i} \phi_{i}(\tau)\right)$ - leads to the effective phase action of the form of interacting quantum rotors

$$
\begin{gathered}
S[\phi]=\int_{0}^{\beta} \mathrm{d} \tau\left\{-\frac{2 t(t z+\bar{\mu})}{U} \sum_{\langle i, j\rangle} \cos \left(\phi_{j}-\phi_{i}\right)\right. \\
\left.+\sum_{i}\left(\frac{\dot{\phi}_{i}^{2}}{2 U}+\mathrm{i} \frac{\bar{\mu}}{U} \dot{\phi}_{i}\right)\right\},
\end{gathered}
$$

where $z$ stands for the coordination number of the lattice. It should be noted that the phase variables are periodic up to $2 \pi$ :

$$
\phi(0)=\phi(\beta)+2 \pi n,
$$

where $n$ is a winding number, which numbers topologically different paths. Following the course of treatment described in detail in [13], we arrive at the critical line equation

$$
1=\frac{1}{2} \int_{-\infty}^{\infty} \mathrm{d} E \frac{\rho(E)}{\sqrt{\frac{t(t z+\bar{\mu})}{U^{2}}[\varepsilon(0)-E]+v^{2}\left(\frac{\mu}{U}\right)}} .
$$

Here $v(x)=x-[x]-1 / 2$ with $[x]$ being a floor function and $\rho(E)$ is a density of states (DOS) defined as:

$$
\rho(E)=\frac{1}{V} \int \mathrm{d} \boldsymbol{k} \delta[E-\varepsilon(\boldsymbol{k})]
$$

where $\varepsilon(\boldsymbol{k})$ is dispersion relation.

\section{Band structure parameters}

Magnetic field affects band structure by splitting and flattening (increasing energy gap-bandwidth ratio) the bands. In lattice system this behaviour is non-systematic and of a fractal nature. In the following we present various scenarios of arbitrary magnetic band structure, that allow us for systematic analysis of the influence of magnetic field on the system properties.

\subsection{Dirac delta bands}

In weak magnetic field presence of the lattice has only minor influence on the properties of the system. Due to this fact, it is useful to consider density of states given by the Dirac deltas which is analogous to the Landau levels. The simplest example consists of equally distributed Dirac delta bands. Critical line Eq. (8) for this case takes form

$$
\begin{aligned}
1 & =\frac{1}{2 n\left|v\left(\frac{\mu}{U}\right)\right|} \\
& +\sum_{i=2}^{n} \frac{1}{2 n \sqrt{\frac{t(t z+\bar{\mu})}{U^{2}}(i-1) E_{g}+v^{2}\left(\frac{\mu}{U}\right)}},
\end{aligned}
$$

where $n$ stands for the number of bands and $E_{g}$ is the energy gap. The first term in Eq. (8) is related to the lowest band. It indicates, for some values of $v(\mu / U)$, that Eq. (8) will not have any solutions, since the second term is non-negative. As a consequence there is a region, where only the Mott insulator exists, located around $\mu / U=0.5+n$. For $n$ bands, each band has an $1 / n$ contribution to the whole DOS. This means that with increasing number of bands, the weight of the lowest band decreases and the MI-only region becomes narrower (see Fig. 1).

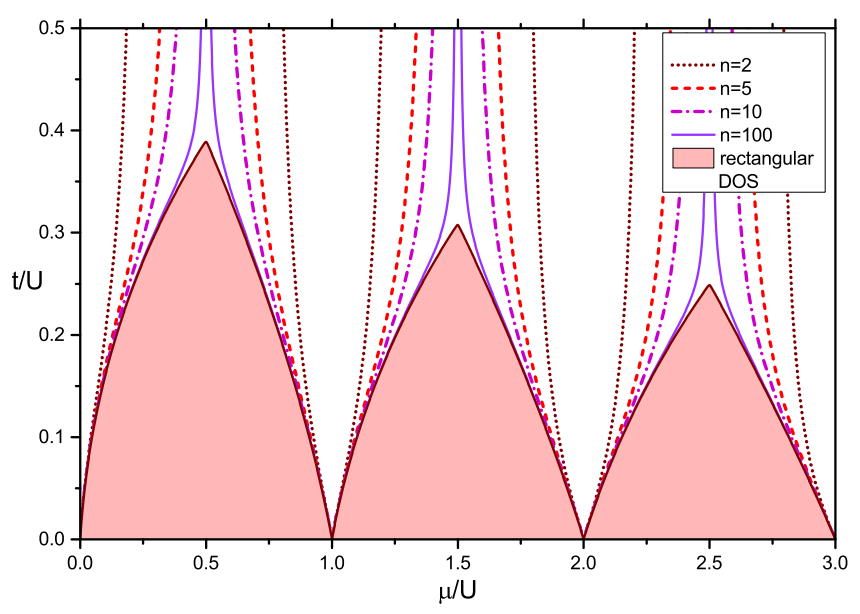

Fig. 1. Phase diagram for $n$ distributed Dirac delta bands. Phase diagram for rectangular DOS is presented for comparison.

\subsection{Rectangular flat bands}

Since bandwidths of the Hofstadter butterfly spectrum are finite, we need to consider their influence on the phase diagram. In order to make the analysis insightful, we use a two-band model. Each band has a rectangular density of states (see Fig. 2). This leads to equation for critical line in the form

$$
\begin{aligned}
1 & =\frac{1}{2 d_{1}} \frac{\sqrt{t(t z+\bar{\mu}) d_{1} / U^{2}+v^{2}(\mu / U)}-|v(\mu / U)|}{t(t z+\bar{\mu}) / U^{2}} \\
& +\frac{1}{2 d_{2}}\left[\frac{\sqrt{t(t z+\bar{\mu})\left(d_{1}+d_{2}+x_{g}\right) / U^{2}+v^{2}(\mu / U)}}{t(t z+\bar{\mu}) / U^{2}}\right. \\
& \left.-\frac{\sqrt{t(t z+\bar{\mu})\left(d_{1}+x_{g}\right) / U^{2}+v^{2}(\mu / U)}}{t(t z+\bar{\mu}) / U^{2}}\right]
\end{aligned}
$$

where $d_{1}$ and $d_{2}$ are widths of the lower and upper band, 


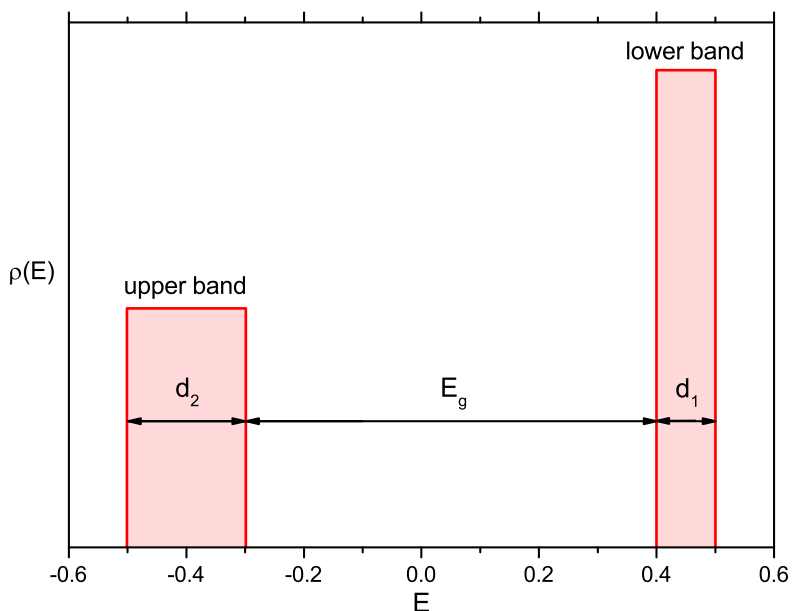

Fig. 2. Example of density of states for the two-band model, with $d_{1}, d_{2}$ being bandwidths of the lower and the upper band, respectively and $E_{g}$ - an energy gap.
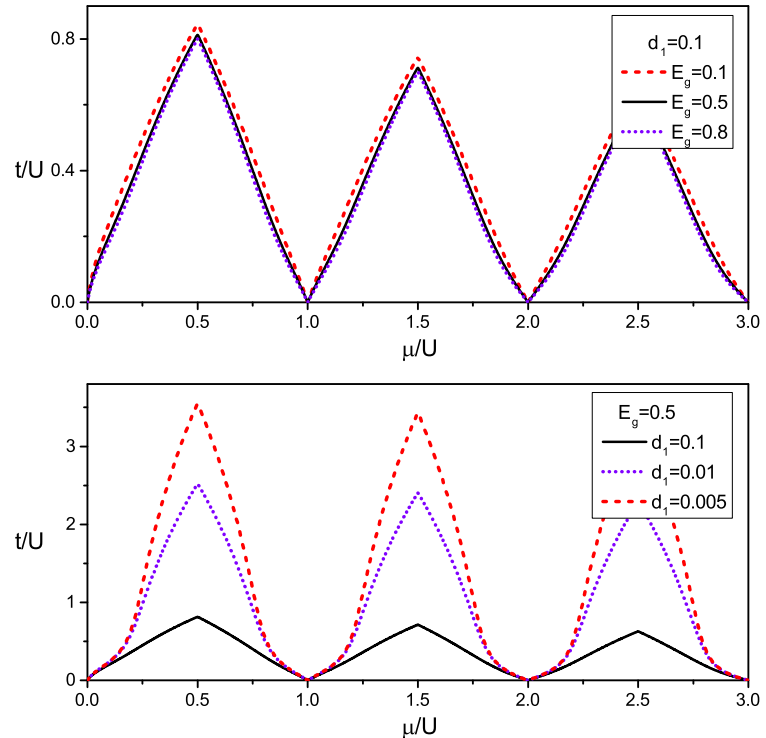

Fig. 3. Phase diagrams for two-band model for (top): constant width of the lower band $d_{1}$ and changing energy gap $E_{g}$, (bottom): constant energy gap and changing width of the lowest band.

respectively and $E_{g}$ is an energy gap separating them. Increasing flatness of the lowest band (defined as $E_{g} / d_{1}$ ) destroys superfluid order (see Fig. 3, top). High flatness of the band translates to the high effective mass, which makes condensation less favourable. This effect is the most pronounced in the vicinity of half-integer values of the chemical potential, where average number of particles per site is close to integer values both in superfluid state as well as in the Mott insulator. On the other hand, increasing energy gap has only minor influence on the phase diagram. For fixed width of the lowest band, increase of energy gap shifts the position of the upper band in which population of bosons is small (see Fig. 3, bottom).

\section{Simulation of magnetic DOS}

The shape of the phase diagram is influenced mainly by the general energy structure (number of bands, bandwidths and energy gaps). The internal structure of the magnetic bands has only minor impact, which is even less important as the number of bands grows. This can greatly simplify the analysis of systems of bosons in synthetic magnetic field. The complicated magnetic densities of states can be replaced by simpler, artificial ones. In order to simulate magnetic densities of states, the following steps must be taken:

- solving Harper's equation [17] at two points in the Brillouin zone: $(0,0)$ and $(\pi / q, \pi / q)$,

- calculation of energy gaps and bandwidths,

- replacing all bands with rectangular density of states preserving calculated bandwidths and gaps.

This procedure becomes more accurate as the number of bands increases although the process becomes slower at $\mu / U=1 / 2+n$ (see Fig. 4). What makes the method more useful is the fact that for the increasing number of bands computational cost of calculating DOS numerically grows very fast. Thanks to this method wide range of magnetic flux values can be considered.
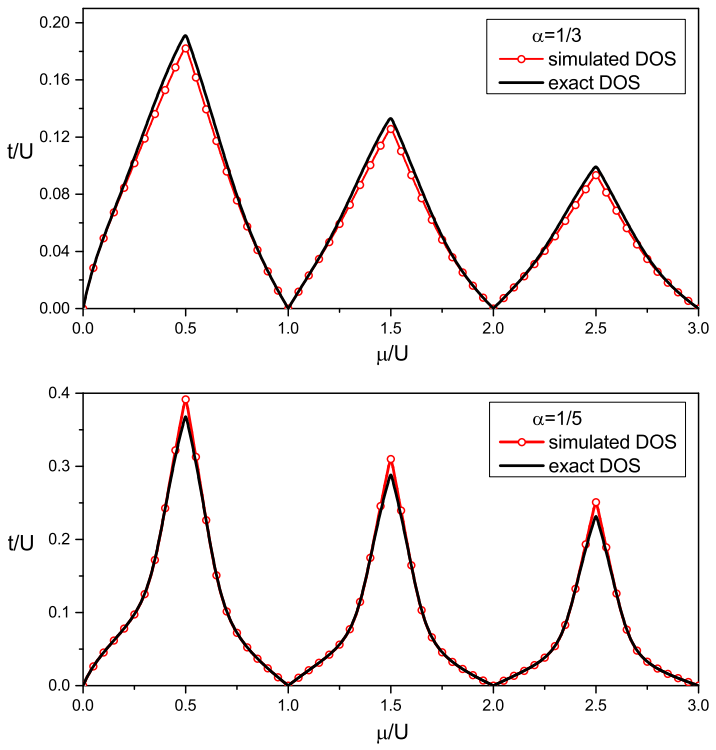

Fig. 4. Phase diagrams for numerical magnetic DOS and simulated DOS. Top: for $\alpha=1 / 3$, bottom: for $\alpha=1 / 5$.

Mainly three factors influence ground state of the Bose-Hubbard model: total bandwidth $(\Delta E)$, number of bands and width of the lowest band. Total bandwidth gives mean-field scaling of critical hopping (see Fig. 5). The other two factors influence the phase diagram in the most pronounced way in the vicinity of $\mu / U=1 / 2+n$ (see Fig. 5). Increasing flatness favours MI state and 

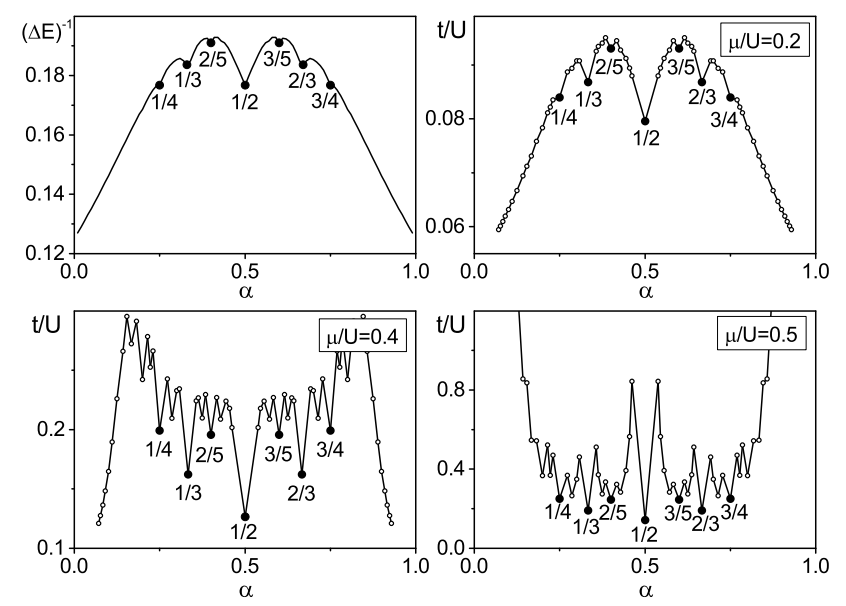

Fig. 5. Critical hopping of the Bose-Hubbard model for a range of magnetic field and chosen values of chemical potential. Inverse of total bandwidth of the Hofstadter butterfly spectrum $(\Delta E)^{-1}$, for comparison.

increasing number of bands narrows the region of rapid increase of critical hopping (corresponding to only-MI region from Sect. 3.1).

\section{Summary}

We have studied properties of the system of bosons in an optical lattice in synthetic magnetic field. In order to determine the ground state of the system we have used the Bose-Hubbard model in the quantum rotor approach. Thanks to this method it was possible to analyze the subtleties of the band structure. Since parameters of the band structure change in non-monotonic way with respect to magnetic field we wanted to analyze it systematically. For this purpose we used two test models: the Dirac delta band model and two-band model. It allowed us to explain the impact of internal energy structure of the Hofstadter butterfly spectrum on the ground state and lead us to a method of simulation of magnetic band structure, which allowed to consider wide range of magnetic fluxes.

\section{References}

[1] D. Pesin, L. Balents, Nat. Phys. 6, 376 (2010).

[2] I. Bloch, J. Dalibard, W. Zwerger, Rev. Mod. Phys. 80, 885 (2008).

[3] M. Greiner, O. Mandel, T. Esslinger, T.W. Hänsch, I. Bloch, Nature 415, 39 (2002).

[4] D. Jaksch, P. Zoller, New J. Phys. 5, 56.1 (2003).

[5] A.R. Kolovsky, EPL 93, 20003 (2011).

[6] M. Aidelsburger, M. Atala, M. Lohse, J.T. Barreiro, B. Paredes, I. Bloch, Phys. Rev. Lett. 111, 185301 (2013).

[7] H. Miyake, G.A. Siviloglou, C.J. Kennedy, W.C. Burton, W. Ketterle, Phys. Rev. Lett. 111, 185302 (2013).

[8] C.J. Kennedy, W.C. Burton, W.C. Chung, W. Ketterle, Nat. Phys. 11, 859 (2015).

[9] J. Struck, M. Weinberg, C. Ölschläger, P. Windpassinger, J. Simonet, K. Sengstock, R. Höppner, P. Hauke, A. Eckardt, M. Lewenstein, L. Mathey, Nat. Phys. 9, 738 (2013).

[10] G. Jotzu, M. Messer, R. Desbuquois, M. Lebrat, T. Uehlinger, D. Greif, T. Esslinger, Nature 515, 237 (2014).

[11] D.R. Hofstadter, Phys. Rev. B 14, 2239 (1976).

[12] T.K. Kopeć, Phys. Rev. B 70, 054518 (2004).

[13] T.P. Polak, T.K. Kopeć, Phys. Rev. B 76, 094503 (2007).

[14] T. Zaleski, T. Kopeć, Phys. Rev. A 84, 053613 (2011).

[15] M.P.A. Fisher, P.B. Weichman, G. Grinstein, D.S. Fisher, Phys. Rev. B 40, 546 (1989).

[16] D. Jaksch, C. Bruder, J.I. Cirac, C.W. Gardiner, P. Zoller, Phys. Rev. Lett. 81, 3108 (1998).

[17] P.G. Harper, Proc. Phys. Soc. A 68, 874 (1955). 